\title{
$p{ }^{6,8}$ He Elastic Scattering at Intermediate Energies
}

\author{
E. Baldini-Neto, B. V. Carlson, R. A. Rego, \\ Departamento de Física, Instituto Tecnológico de Aeronáutica, Centro Técnico Aeroespacial, \\ 12228-900, São José dos Campos, São Paulo, Brazil \\ and M. S. Hussein \\ Instituto de Física, Universidade de São Paulo, 05508-900, São Paulo, Brazil
}

Received on 12 September, 2003

\begin{abstract}
Using a relativistic nuclear optical potential consisting of a Lorentz scalar, $V_{s}$, and the time-like component of a four-vector potential, $V_{0}$, with a Gaussian form factor, we have calculated elastic scattering differential cross sections and polarizations for $p+{ }^{6,8} \mathrm{He}$ at intermediate energies. We compare our results with those of the Relativisitic Impulse Approximation (RIA) and with the available experimental data in the range of $0 \leq t \leq 0.22(\mathrm{GeV} / \mathrm{c})^{2}$.
\end{abstract}

\section{Introduction}

With the advent of radioactive nuclear beams and the discovery that nuclear matter, under certain conditions, may present a halo structure, a renewed interest has surged in the investigation of sizes and radial shapes of nuclei. In the case of light neutron-rich nuclei, this new halo structure is composed of an extended low-density distribution of loosely bound valence neutrons (halo) surrounding a core consisting of the majority of the nucleons.

From the experimental point of view, the study of proton-nucleus scattering at intermediate energies is a useful method for determining accurate nuclear matter distributions in stable nuclei $[1,2,3]$ mainly due to the fact that the Glauber multiple-scattering formalism can correlate the differential cross sections and the matter distribution [2]. Recently, it has also been used extensively to determine the extension of the nuclear matter density of exotic nuclei. In particular, in a recent set of experiments, performed at GSI, inverse kinematics were used to study the elastic scattering of $p+{ }^{4,6,8} \mathrm{He}$ and determine their matter densities [4, 5, 6]. On the theoretical side, elastic scattering at intermediate energies can be calculated using either non-relativistic approaches as done in Refs. [7, 8], or through relativistic models $[9,10]$, usually with about the same good results. An advantage of a relativistic optical model is that its two potentials simultaneously determine both the central and spinorbit interactions. When the relativistic impulse approximation (RIA) is used [11, 12], these interactions may be determined in terms of the corresponding scalar and vector nuclear densities [14].

In a previous work [15] we have used a relativistic nuclear optical potential, constructed from a Lorentz scalar, $V_{s}$, and the time-like component of the four-vector potential, $V_{0}$, with Gaussian form factors to calculate elastic scattering angular distributions and analyzing powers for $p+{ }^{4} \mathrm{He}$ at intermediate energies, comparing the results with a RIA calculation. Motivated by the quality of our results, we have extended the optical potential parameters obtained in that case in order to calculate angular distributions and polarizations for the elastic scattering of $p+{ }^{6,8} \mathrm{He}$. We also compare our results with those of a RIA calculation, as well as with the very recent experimental data of angular distributions measured at values of the momentum transfer up to 0.22 $(\mathrm{GeV} / \mathrm{c})^{2}[13]$.

\section{The Dirac optical potential}

In relativistic optical model analysis of intermediate energy scattering [9], the Dirac equation is usually used in the form,

$$
\left\{\vec{\alpha} \cdot \vec{p}+\beta\left[m+V_{s}(r)\right]+\left[V_{0}(r)+V_{c}(r)\right]\right\} \Psi(\vec{r})=E \Psi(\vec{r}),
$$

where $V_{s}$ is an attractive Lorentz scalar potential, $V_{0}$ is the repulsive time-like component of a four-vector potential and $V_{c}$ is the Coulomb potential. The choice of the potentials is motivated by meson exchange considerations and simplicity. The simplest meson exchange interaction possessing a certain justification on physical grounds, which is also capable of providing nuclear saturation properties, takes into account the exchange of an attractive intermediate range isoscalar scalar meson and a repulsive shorter range isoscalar vector meson $[14,16,17,18]$. The two corresponding potentials $V_{s}$ and $V_{0}$ play an essential role in the description of both elastic scattering and polarization data at intermediate energies since their sum is the principal contribution to the central potential while their difference determines the spin-orbit interaction [19]. The scalar (vector) optical potentials used in the analyses here can be written as

$$
\begin{aligned}
& V_{s}=U_{s} f_{U_{s}}(r)+\imath W_{s} f_{W_{s}}(r) \\
& V_{0}=U_{0} f_{U_{0}}(r)+\imath W_{0} f_{W_{0}}(r),
\end{aligned}
$$


with each of the potentials possessing both real and imaginary parts with possibly different radial dependences.

In the late seventies, Arnold, Clark and Mercer [9] measured angular distributions and polarizations for $p+{ }^{4} \mathrm{He}$ elastic scattering at intermediate energies, from 0.561 to $1.73 \mathrm{GeV}$. With a modified Woods-Saxon parametrization for the Dirac optical potential, with different sets of parameters at each energy, they obtained very impressive fits to the experimental data. However, nuclear structure calculations demonstrate that the nuclear densities of ${ }^{4} \mathrm{He}$ have a radial dependence $f(r)$ which is very nearly Gaussian. Based on this physical evidence we have therefore chosen a Gaussian parametrization of the form factor [15] to fit the experimental data for $p+{ }^{4} \mathrm{He}$ elastic scattering at intermediate energies and we have restricted our attention to energies near those at which experimental data for $p+{ }^{6,8} \mathrm{He}$ elastic scattering exists, viewing an extrapolation of our global parameters to these latter cases. In what follows we will discuss our fitting procedure which is different from that of Ref. [9].

\section{Results}

\section{1 $p+{ }^{6,8}$ He elastic scattering}

We consider the elastic scattering of $p+{ }^{6,8} \mathrm{He}$ at the laboratory energies for which data exist [5, 13], $E_{L a b}=0.717$ $\mathrm{GeV}$ and $E_{L a b}=0.671 \mathrm{GeV}$, respectively. To perform the RIA calculation for ${ }^{6,8} \mathrm{He}$, we followed the same procedure used for ${ }^{4} \mathrm{He}$ in Ref. [15]. We generated equal scalar and vector matter densities using the appropriate Gaussian harmonic oscillator (HO) geometrical parameters of Ref. [5], together with the relativistic Love-Franey NN t-matrix of Ref. [21]. The results of the RIA calculation (full lines) for the angular distributions, shown in Figs. (3) and (4), agree well with the experimental data in the range of very low values of the momentum transfer but, as this quantity increases, they deviate from the experimental data in form and absolute values. These results are very similar to those obtained for $p+{ }^{4} \mathrm{He}$ elastic scattering.

We have attempted to extend our fit of the ${ }^{4} \mathrm{He}$ data [15] by interpreting them in the context of a simple RIA calculation. To do so, we extract the effective strengths, $\tilde{V}_{0 i}=\frac{1}{4}\left(U_{i}, W_{i}\right)\left[\pi r_{0, i}^{2}\right]^{3 / 2}$, that, when multiplied by the ${ }^{4} \mathrm{He}$ density, would yield the potentials obtained in our fit to the $p+{ }^{4} \mathrm{He}$ data. We have multiplied these strengths by the appropriate ${ }^{6,8} \mathrm{He}$ densities, based on the occupation of the $1 s_{1 / 2}$ and $1 p_{3 / 2}$ orbitals, obtained using the Gaussian (HO) parametrization of Ref. [5], to obtain effective Dirac potentials for these systems,

$$
\begin{aligned}
V_{i}(r) & =\tilde{V}_{0 i}\left(\frac{4}{\left(\pi r_{0 c}^{2}\right)^{3 / 2}} \exp \left[-r^{2} / r_{0 c}^{2}\right]\right. \\
& \left.+\zeta \frac{2}{3} \frac{1}{\left(\pi r_{0 v}^{2}\right)^{3 / 2}} \frac{r^{2}}{r_{0 v}^{2}} \exp \left[-r^{2} / r_{0 v}^{2}\right]\right)
\end{aligned}
$$

where $\zeta=2,4$ for ${ }^{6} \mathrm{He}$ and ${ }^{8} \mathrm{He}$, respectively, with $r_{0 c}$ being the core $\left(1 s_{1 / 2}\right)$ radial parameter and $r_{0 v}$ the valence $\left(1 p_{3 / 2}\right)$ one.
TABLE I. Calculated rms radii in ${ }^{4} \mathrm{He},{ }^{6} \mathrm{He}$ and ${ }^{8} \mathrm{He}$ compared with the experimental values taken from Refs. [5, 13], labeled with indices (a) and (b) respectively.

\begin{tabular}{rrrr} 
& $r_{r m s}(\mathrm{fm})$ & $r_{r m s_{(a)}}(\mathrm{fm})$ & $r_{r m s_{(b)}}(\mathrm{fm})$ \\
\hline${ }^{4} \mathrm{He}$ & 1.50 & $1.49(3)$ & - \\
${ }^{6} \mathrm{He}$ & 2.36 & $2.30(7)$ & $2.38 \pm 0.05$ \\
${ }^{8} \mathrm{He}$ & 2.49 & $2.45(7)$ & $2.41 \pm 0.04$ \\
\hline
\end{tabular}

The radii were modified slightly to take into account the differences between the experimental ${ }^{4} \mathrm{He}$ radius of Ref. [5] and those obtained in our previous fit. In order to check our extrapolations we have used the generated potentials to calculate the effective rms radii of both systems. As we can see from Table I there is a resonable agreement with the experimental values taken from Refs. $[5,13]$ in the case of ${ }^{6} \mathrm{He}$. The resulting angular distribution is shown in Fig. 1 and is also in good agreement with the available experimental data $[5,13]$, taking into account the large error bars at higher values of the momentum transfer, given in Ref.[13]. We have also performed a free fit (dotted lines) of the data, taking as a first guess the physical parameters of Ref. [15], in order to measure the quality of our physical extrapolation. As shown in Fig. 1, the free fit (dotted lines) provides slightly better agreement with the experimental data when compared to the physical extrapolation. In the case of $p+{ }^{8} \mathrm{He}$ elastic scattering, the physical extrapolation provides only slightly better agreement with the data than the RIA results, both shown in Fig. 2. This is also reflected in a poorer reproduction of the rms radius, given in Table I. Here, we have again performed a free fit of the parameters which yields fairly good agreement with the experimental data. In Figs. 3 and 4 we compare the polarizations for ${ }^{6} \mathrm{He}$ and ${ }^{8} \mathrm{He}$, calculated with the physical extrapolation, with the RIA results. Substantial differences exist between the two, as was the case in $p+{ }^{4} \mathrm{He}$ scattering [15]. At present no experimental data exist for the polarizations. Although extremely difficult to perform, experimental measurements of these polarizations would be useful for better determining their optical potentials.

\section{Conclusions}

We have calculated $p+{ }^{6,8} \mathrm{He}$ elastic scattering differential cross sections and polarizations using the relativistic impulse approximation and an adjusted Dirac optical potential. We have shown that the RIA results obtained using the parameters of Ref. [5] describe both systems well at low values of the momentum transfer. However, they deviate substantially from the data at higher momentum transfer and, moreover do not reproduce the expected oscillatory structure in the angular distributions and the corresponding polarizations. As an alternative to the RIA, we have used physical motivated Gaussian form factors given in the fit of the $p+{ }^{4} \mathrm{He}$ data [15] and, then attempted to extend the parameters obtained to the cases of $p+{ }^{6,8} \mathrm{He}$, with quite resonable results in the first case and poorer agreement in the second 


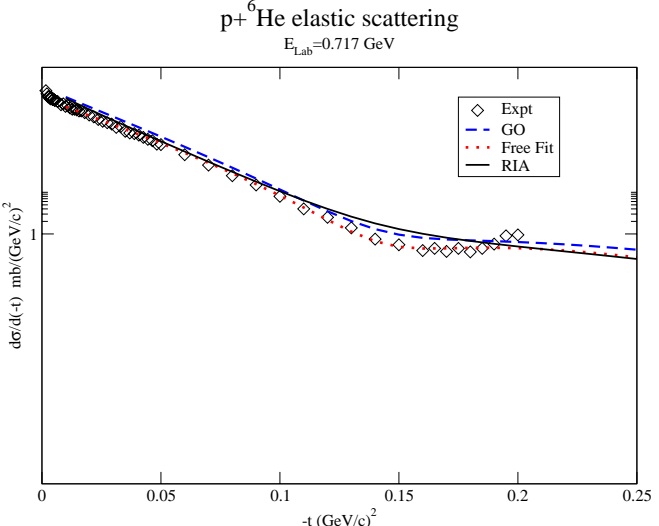

Figure 1. Differential cross section of $p+{ }^{6} \mathrm{He}$ at $E_{L a b}=0.717$ $\mathrm{GeV}$. The dashed line corresponds to our calculation, the full line corresponds to the RIA calculation while the dotted line stands for a free fit to the experimental data (diamonds) from Refs. [5, 13]. Where not shown the experimental error are within the diamonds.

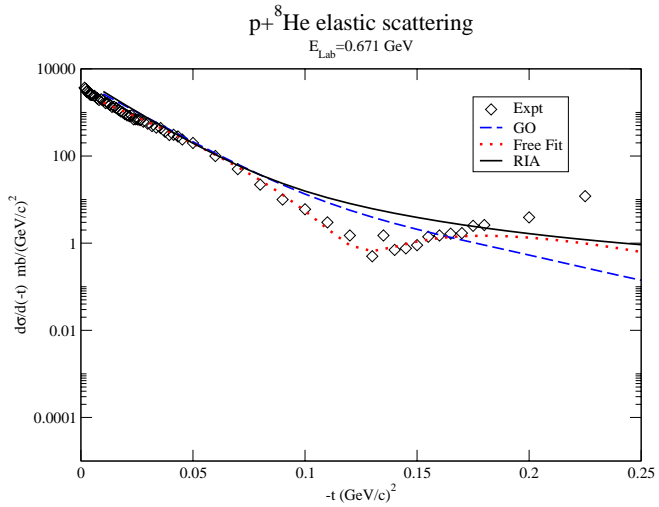

Figure 2. Differential croos section of $p+{ }^{8} \mathrm{He}$ at $E_{L a b}=0.671$ $\mathrm{GeV}$. The dashed line corresponds to our calculation, the full line corresponds to the RIA calculation while the dotted line stands for a free fit to the experimental data (diamonds) from Refs. [5, 13]. Where not shown the experimental errors are within the diamonds.

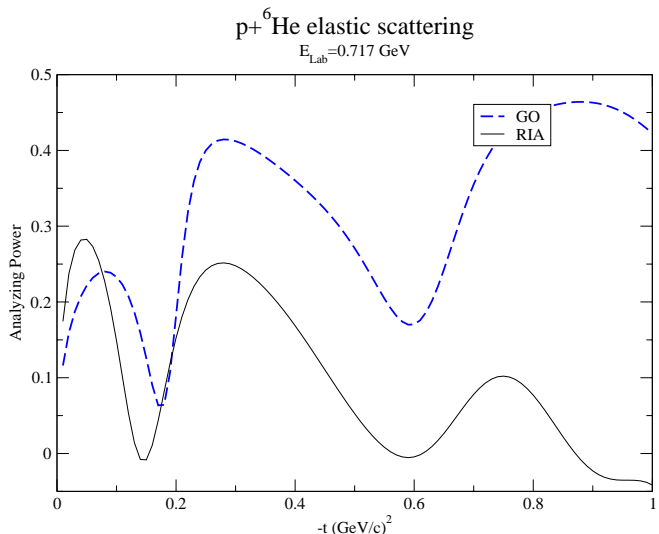

Figure 3. Polarization for $p+{ }^{6} \mathrm{He}$ at $E_{L a b}=0.717 \mathrm{GeV}$. The dashed line corresponds to the physical extrapolation while the full line corresponds to the RIA calculation.

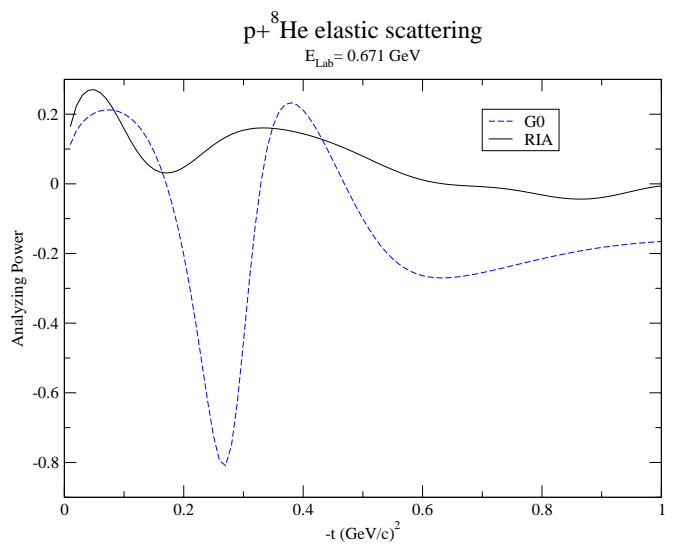

Figure 4. Polarization for $p+{ }^{8} \mathrm{He}$ at $E_{L a b}=0.671 \mathrm{GeV}$. The dashed line corresponds to the physical extrapolation while the full line corresponds to the RIA calculation.

one. In all cases, we obtained reasonable fits to the data where the parameters were permited to vary freely. We have also calculated the polarization in $p+{ }^{6,8} \mathrm{He}$ scattering. As in the case of $p+{ }^{4} \mathrm{He}$, we find large differences between the RIA results and those of our physical extrapolation. Experimental data, which do not exist at present, will be necessary to resolve these discrepancies.

In closing, we should mention that a possible reason for the failure of the RIA in describing the $p+{ }^{6,8} \mathrm{He}$ data, mainly at higher momentum transfer, is that it has been pushed beyond the limit of its applicability in the simple manner in which it is used here. The decomposition of the effective Dirac potential into a scalar potential and the fourth component of vector is valid in the target rest frame. The scattering calculations, however, are performed in the CM frame and the boost that carries one frame to the other will convert the vector fourth component potential into a full vector potential. For proton scattering on a system such as ${ }^{40} \mathrm{Ca}$, the vector components introduced are small and can be neglected. For extremely light systems, such as those studied here, this may not be the case. We plan to examine the importance of this effect in the future.

\section{Acknowledgments}

The authors would like to thank FAPESP, Fundação de Amparo à Pesquisa do Estado de São Paulo, for financial support. B. V. Carlson and M. S. Hussein ackowledge support from the CNPq, Conselho Nacional de Desenvolvimento Científico e Tecnológico. EBN would like to thank N. Alamanos for providing the recent experimental data.

\section{References}

[1] I. Tanihata et al., Phys. Rev. Lett. 55, 2676 (1985).

[2] G. D. Alkhazov et al., Phys. Rep. C 42, 89 (1978).

[3] J. W. Negele et al., Adv. Nucl. Phys. 19, 1 (1989).

[4] G. D. Alkhazov et al., Phys. Rev. Lett 78, 2313 (1997).

[5] P. Egelhof et al., Eur. Phys. J. A. 15, 27 (2002).

[6] S. R. Neumaier et al., Nucl. Phys. A 712, 247 (2002). 
[7] J. S. Al-Khalili and J. A. Tostevin, Phys. Rev.C 57, 1846 (1998).

[8] K. Amos, S. Karataglidis, and P. K. Deb, nucl-th/0202081, (2002).

[9] L. G. Arnold, B. C. Clark, and R. L. Mercer, Phys. Rev. C 19, 917 (1979)

[10] L. G. Arnold, B. C. Clark, R. L. Mercer, and P. Schwandt, Phys. Rev.C 23, 1949 (1981)

[11] J. A. McNeil, J. R. Shepard, and S. J. Wallace, Phys. Rev. Lett. 50, 1439, 1443 (1983).

[12] B. C. Clark, S. Hama, R. L. Mercer, L. Ray, and B. D. Serot, Phys. Rev. Lett. 50, 1664 (1983).

[13] F. Aksouh, $P h D$. Thesis, "Investigation of the core-halo structure of the neutron rich nuclei ${ }^{6,8} \mathrm{He}$ by intermediate-energy elastic proton scattering at high momentum transfer", (2002); P. Egelhof, "Invited talk presented at the International Sym- posium on Physics of Unstable Nuclei", Halong-Bay (Vietnam), November, 20-25 (2002) to appear in Nucl. Phys. A

[14] B. D. Serot and J. D. Walecka, Advances in Nuclear Physics, "The Relativistic Many-Problem", Plenun Press - New York (1986).

[15] E. Baldini-Neto, B. V. Carlson, R. A. Rego, and M. S. Hussein, Nucl. Phys. 724345 (2003).

[16] K. Erkelenz, Phys. Rep. C 13, 194 (1974).

[17] G. Brown and D. Jackson, The nucleon-nucleon interaction, (North-Holland-Amsterdam), 1976.

[18] T. Ueda, F. E. Riewe, and A. E. S. Grenn, Phys. Rev. C 17, 1763 (1978)

[19] W. H. Furry, Phys. Rev. 50, 784 (1936).

[20] B. V. Carlson and D. Hirata, Phys. Rev. C 62, 054310 (2000).

[21] C. J. Horowitz, Phys. Rev. C 31, 1340 (1985). 